\title{
Penetrating Keratoplasty and Cataract Surgery: The Advantages of an Extracapsular Technique Combined with Posterior Chamber Intraocular Implantation
}

\author{
C. M. KIRKṆESS, P. Y. Y. CHEONG and A. D. McG. STEELE \\ London
}

\begin{abstract}
Summary
The results of 28 consecutive triple procedures and 33 cases of combined penetrating keratoplasty and extracapsular extraction are presented. All the eyes with the triple procedure maintained a clear graft; 77 per cent achieved an acuity of $6 / 12$ or better. The average time before a refractive correction could be dispensed was 8.2 months, while after a combined procedure the interval was 17.4 months, although in both groups the average time until the best visual acuity was reached was similar.
\end{abstract}

Cataract may occasionally be associated with corneal disease. At one time, it was considered that penetrating keratoplasty and cataract extraction could not be combined in one operation because of the risk of complications from such surgery. Katzin ${ }^{1}$ and later Casey² reported that cataract could be removed safely at the same time as keratoplasty was performed. Kaufman ${ }^{3}$ then suggested that any significant cataract should be removed because of the likelihood that opacification would increase following keratoplasty and that cataract surgery subsequent to grafting may prejudice graft survival.

As cataract techniques have become more sophisticated, each advance in technique has been adapted by corneal surgeons to a combined procedure with keratoplasty. Probably the most significant advance in cataract surgery was the introduction of extracapsular extraction which greatly reduced the incidence of aphakic retinal detachment and aphakic glaucoma. ${ }^{4.5 .6}$ The advent of more satisfactory intraocular lens designs encour- aged some surgeons to combine intraocular implantation with keratoplasty ${ }^{7}$ but it was not until posterior chamber lenses were re-introduced $^{8.9}$ and viscoelastic materials were developed $^{10}$ that a 'triple procedure'-extracapsular extraction, posterior chamber intraocular lens implantation and penetrating keratoplasty could be practised widely and safely.

This study reports the results of two groups of patients undergoing penetrating keratoplasty and cataract surgery. The first group were treated with the triple procedure, the second underwent penetrating keratoplasty and extracapsular extraction without implantation.

\section{Material and Methods}

Patients undergoing combined surgery for cataract and corneal disease under the care of the surgeons of the Corneal Clinic. Moorfields Eye Hospital during the period $1 / 1 / 81$ to $31 / 12 / 85$ were identified. 


\section{Triple procedure}

Preoperative assessment included measurement of axial length by ultrasonic A scan, keratometry where possible and assessment of the intraocular lens (IOL) power using the Sanders-Retzlaf-Kraff" regression formula, empirically, without making any allowance for the possible change in average keratometry after corneal grafting. In 8 cases, the keratometry could not be assessed due to excessive corneal irregularity.

All surgical procedures were performed in a similar fashion under general anaesthesia. The anterior segment was supported using an anterior scleral ring. Following preparation of the host bed, an anterior capsulectomy was performed using fine intraocular scissors; the nucleus was dislocated and expressed; the remaining cortical fibres were removed using a Pearce/McIntyre infusion and aspiration canula. Healonid or HMPC 2 per cent was then instilled into the capsular bag and a posterior chamber lens, with loops angled forwards at 10 degrees, was inserted into the bag. If necessary, the pupil was constricted with intraocular miochol. The anterior chamber was reformed with viscoelastic material. The donor cornea was either prepared from a whole eye or from a corneoscleral disc stored in MK medium. The donor cornea was first secured with a $7 / 0$ cross-over silk suture and then 4 . cardinal $10 / 0$ nylon sutures. The graft was sutured in place with a continuous $10 / 0$ nylon suture and the interrupted sutures were removed. Alternatively interrupted sutures alone were used or in a few cases a combination of interrupted and continuous sutures was employed.

\section{Combined procedure}

For the combined procedure the technique was similar except that no lens was inserted

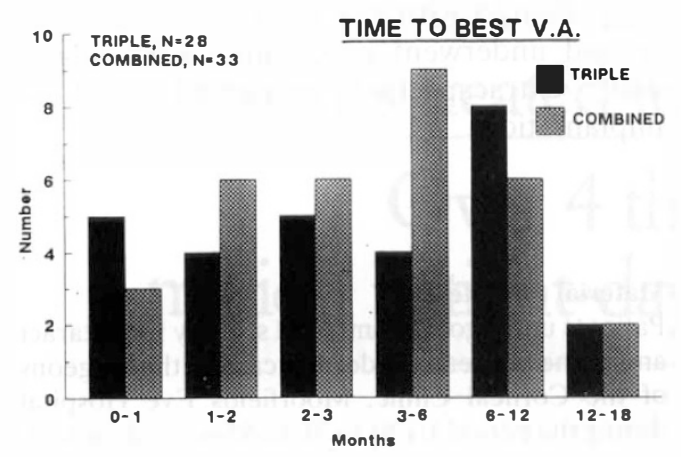

Fig. 1. The time taken to best visual acuity. The first date when the patient with full refractive correction reached his best and most stable acuity after surgery is taken. and usually a peripheral iridectomy was performed.

In all cases subconjunctival steroids and gentamycin were given at the end of the procedure and postoperatively topical prednisolone drops in reducing doses, antibiotic drops and a cycloplegic were given.

\section{Results \\ Triple procedure}

Twenty nine consecutive cases were identified during the study period. One patient died in the peri-operative period and is excluded. Follow-up varied from a minimum of 16 months, to six years, mean 28.4 months. Age at the time of surgery ranged from 38-89 years. There were nine males and nineteen females. During the study two females underwent surgery to both eyes, the surgery for each eye being separated by about 12 months.

Indications for keratoplasty included Fuch's endothelial dystrophy in 50 per cent of cases; interstitial keratitis, 11 per cent; graft failure, 11 per cent; keratoconus, 7 per cent; and other corneal dystrophies, 7 per cent. All patients had significant lens opacities. Graft sizes ranged from $7.0-8.5 \mathrm{~mm}$, the majority $7.5 \mathrm{~mm}$. Most (68 per cent) donors were the same size as the host bed (this figure also includes those donors punched from a corneoscleral disc which from necessity must be oversized by at least $0.5 \mathrm{~mm}$ ), while the remainder, all cut from whole eyes, were oversized by $0.5 \mathrm{~mm}$ trephine size. All the grafts in this group have remained clear and 77 per cent have achieved a corrected visual acuity of $6 / 12$ or better. Two patients had an acuity of $6 / 60$ and the remainder between $6 / 18$ and $6 / 36$. Vision was poor in 23 per cent $(6$ patients) for the following reasons: senile macular degeneration -3 , lamellar macular hole -1 , optic atrophy -1 , and in one case accurate acuity could not be determined in an educationally subnormal patient, who appeared to show very substantial improvement in visual function. The best visual acuity was achieved within 3 months by 51 per cent of patients. The mean time to best achievable acuity was 5.1 months. (Figs 1 and 2).

\section{Combined procedure}

Over the same period of time, 35 combined 


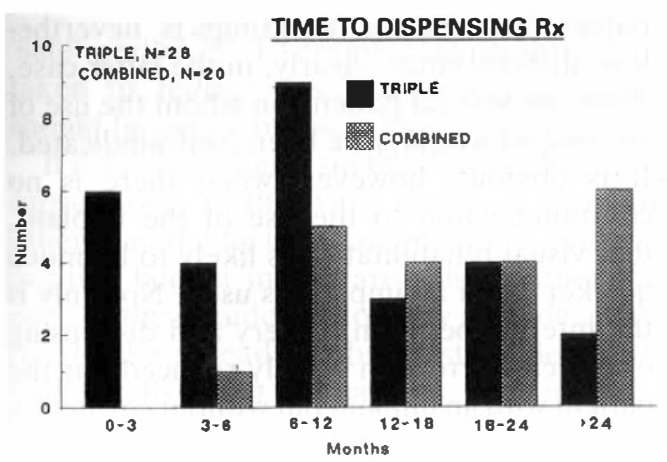

Fig. 2. The time to dispensing $R x$. The time taken from the time of surgery until an optical correction, either glasses or contact lens could be dispensed to the patient is shown.

procedures were carried out but 2 cases were lost to follow up in the immediate post-operative period and are excluded. Follow-up was over the same period as for the triple procedure but the mean follow up time was 36.1 months. Age at surgery was from 35 to 89 years. There were 11 males and 22 females. Indications for keratoplasty included Fuch's endothelial dystrophy, 30 per cent; interstitial keratitis, 15 per cent; graft failure, 15 per cent; keratoconus, 6 per cent and a diverse group, 33 per cent. In 21 per cent ( 7 patients), there could be considered an absolute contraindication to implantation; 4 had herpetic keratouveitis, including one regraft; in 1 there was a perforated corneal ulcer and in 2 cases there was a history of anterior uveitis. Relative and practical contraindications to intraocular lens implantation included high myopia, and aphakia without pseudophakos in the fellow eye but in 20 cases there was no absolute contraindication to implantation. Just over half ( 54 per cent) had a donor of the same size the remainder being oversized by $0.5 \mathrm{~mm}$; 66 per cent were $7.5 \mathrm{~mm}$, there was one $8.5 \mathrm{~mm}$ and the rest were $8.0 \mathrm{~mm}$.

In this group only 57 per cent obtained an acuity of $6 / 12$ or better. All but 2 of these grafts remained clear. Of the 2 that failed, one has since been successfully regrafted. One patient had an acuity of counting fingers, while the remainder had visual acuities between 6/18 and 6/36. Best acuity (Fig. 1) was achieved by 50 per cent of the eyes by 4 months after surgery with a mean of 5 months but useful vision was not achieved until a mean period of 15.6 months postoperatively, that is until a contact lens or spectacles could be dispensed.

The subgroup (20 eyes) of the combined group in whom there was no absolute contraindication to implantation were compared with the triple group from the point of view of the time the patients had to wait until a correction for refractive error could be dispensed, usually spectacles in the case of the triple group and either spectacles or contact lenses in the combined group. Although the time to best achievable visual acuity did not vary significantly in either group, the triple group received a stable optical correction earlier than the combined group, at a mean of 8.2 months compared to 17.4 months for the combined subgroup. (Fig. 2).

The deviation from projected refractive error, usually emmetropia, for the triple group, is shown in Figure 3. Fifty per cent had a spherical equivalent refractive error of 3 dioptres or less.

\section{Complications}

Triple group

Rejection episodes occurred in 4 patients, all before 12 months, all resolved on treatment leaving a clear graft and unaltered visual acuity. Raised intraocular pressure was found in the immediate post-operative in 3 cases, a late rise in pressure was seen in 2 more cases but only one patient required medication

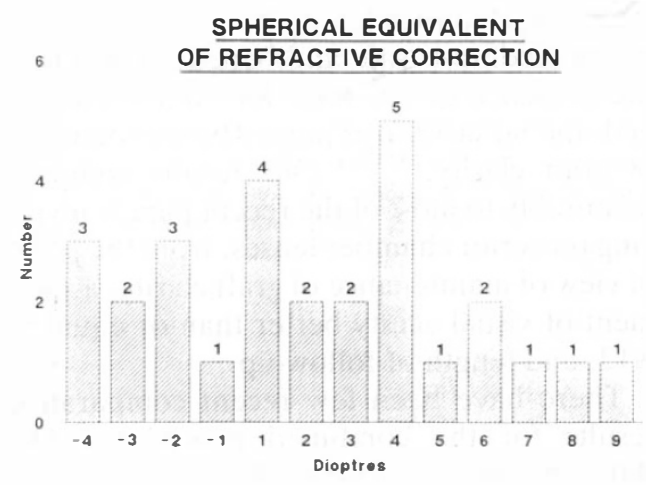

Fig. 3. The refractive error for the patients undergoing the triple procedure was measured and its deviation from the desired and predicted refractive error (usually emmetropia) is recorded in dioptres of spherical equivalents 
(timolol) for long term control of the pressure. Posterior synechiae to the pseudophakos was found in 3 patients. Posterior capsulotomy was performed using $\mathrm{Nd}$ : Yag laser on one patient at 9 months. Anisometropia due to unpredicted refractive error was found in 2 cases and intolerable astigmatism was seen in one eye.

\section{Combined group}

This group had a 30 per cent incidence of graft rejection episodes, half occurring within the first 12 months the rest occurring up to 3 years later. Two eyes had multiple rejection episodes. These grafts decompensated and regrafting was performed. All others remained clear.

Five eyes had persistently raised intraocular pressure beyond the immediate postoperative period requiring therapy and one of these required multiple surgical procedures to control the intraocular pressure.

Eight patients had posterior capsular thickening requiring $\mathrm{Nd}$ : Yag laser capsulotomy.

Recurrence of herpetic keratitis was seen in four patients.

Eleven patients had associated retinal or optic disc problems preventing good visual acuity, including one retinal detachment, successfully reattached, occurring after the graft surgery.

\section{Discussion}

There have been many reports of the triple procedure involving both iridocapsular and posterior chamber lenses which suggest that the procedure is safe and beneficial and that the presence of the lens does not prejudice endothelial survival as judged by maintenance of graft clarity. ${ }^{7.12 .13 .14}$ Our results compare favourably to most of the recent papers involving posterior chamber lenses, from the point of view of maintenance of graft clarity, attainment of visual acuity better than or equal to $6 / 12$, and length of follow-up.

There have been few recent comparative results for the combined procedure. The difference in our average follow up may partly reffect our increasing awareness that the triple procedure was safe and the consequent willingness to undertake the procedure when there was no contraindication. Direct com- parison between the two groups is, nevertheless, difficult since, clearly, in the latter case, there are several patients in whom the use of an implant would have been contraindicated. It is obvious, however, when there is no contraindication to the use of the implant, that visual rehabilitation is likely to be much quicker when an implant is used. Not only is the interval between surgery and dispensing of optical correction greatly reduced but the patient with an implant but without correction is already visually at a much greater advantage than a similar patient who has undergone the combined procedure since the latter is also aphakic. The major difference between these groups is a result of the time interval that is required for the fitting of contact lens following surgery. Most contact lens fitters prefer the graft suture to have been removed before a contact lens is fitted. The incidence of complications in the combined group also compares favourably to earlier published series. Given that many cases were complex, the results confirm that the technique may be undertaken when an implant is contraindicated, but where there is coexistent cataract and corneal disease. It is our opinion, therefore, that in the absence of a positive contraindication, such as grafting 'a chaud', that a posterior chamber intraocular lens should be used when undertaking combined corneal and cataract surgery.

The time taken for most eyes undergoing a triple procedure to achieve their maximum visual potential is considerably longer than would be expected from similar aged patients undergoing cataract surgery alone. Some influence may be expected from the time it takes a graft to recover normal thickness and clarity after keratoplasty but almost certainly some effect may be due to the influence of peroperative macular phototoxicity from the coaxial illumination of the operating microscope. Recent reports have implicated the length of time taken for wound closure following implantation as being an important feature in light induced macular damage, because this is the period when light may be focussed on the macula by the implant. ${ }^{15}$ In cataract surgery this period is relatively short but in keratoplasty the time may be three or four-fold greater and may well exceed the 
'safe' period. ${ }^{16.17}$ Every precaution should be taken to reduce this hazard and surgeons should be aware of the risks.

It is difficult to explain the reduced need for capsulotomy in the triple group on grounds other than the fact that an implant provides a positive benefit in this area also. Either the edge of the pseudophakos may provide a barrier to the spread of fibroblasts or lens epithelial cells along the capsule, or it may, by constant movement against the capsule, gently abrade it so that any ingrowing cells are removed.

The benefit of power calculation of intraocular lenses in reducing unacceptable postoperative degrees of ametropia is widely accepted. Binder ${ }^{13}$ has demonstrated that approximately 50 per cent of eyes in his series of triple procedure have less than 2 dioptres of refractive error, a figure similar to our own. By applying an updated $\mathrm{A}$ constant to the standard SRK formula, ${ }^{11} 91$ per cent of the eyes in his series would have been brought within 2 dioptres of emmetropia. This would have the added benefit of bringing a larger number of patients into a useful level of uncorrected visual acuity.

The triple procedure offers patients the chance of quicker rehabilitation after graft and cataract surgery with little risk of complications.

This paper was presented at the OSUK as a video. We are most grateful to $\mathrm{Mr}$ Alan Lacey for his invaluable work and skill in preparing the videotape and graphics.

\section{References}

${ }^{1}$ Katzin HM and Meltzer JF: Combined surgery for corneal transplantation and cataract extraction. Am J Ophthalmol 1966, 62: 556-60.

${ }^{2}$ Casey TA: The combined operation of cataract and corneal graft. Trans Ophthalmol Soc UK 1969, 89: 659-68.

${ }^{3}$ Kaufman HE: Combined graft and cataract extraction. Am J Ophthalmol 1974, 77: 824-9.

${ }^{4}$ Emery JM and McIntyre DJ: Extracapsular Cataract Surgery, St Louis, CV Mosby 1983, 337-64.

${ }^{5}$ Hyde LL: Extracapsular cataract extraction combined with corneal grafting in Emery $J$ (ed) Current Concepts of Cataract Surgery: Selected Proceedings of the Fourth Bienniel Cataract Surgical Congress. St Louis, CV Mosby Co 1976; 162-6.

${ }^{6}$ Polack FM: Corneal Transplantation. New York, Grune \& Stratton, 1978; 149-60.

${ }^{7}$ Hunkler J, Hyde LL: The triple procedure: combined penetrating keratoplasty, cataract extraction and lens implantation. Am Intra-ocular Implant Soc J 1979, 5: 222-4.

* Pearce JL: A new sutured posterior chamber intraocular lens. Trans Ophthalmol Soc UK 1976, 96: 6-10.

${ }^{9}$ Shearing SP: A practical posterior chamber lens. Contact and Intraocular Lens Med J 1978, 4: 114-9.

11) Steele ADMcG: Visco-elastic materials in keratoplasty. Trans Ophthalmol Soc UK 1983, 103: 268-9.

1 Retzlaff J, Sanders DR, Kraff MC: A Manual of implant power calculation: SRK formula. Oregon, Medford 1981.

12 Lindstrom RL, Harris WS, Doughman DJ: Combined penetrating keratoplasty, extracapsular cataract extraction, and posterior chamber lens implantation. Am Intra-ocular Implant Soc J 1981, 7: 130-2.

${ }_{13}^{13}$ Binder PS: Intraocular lens powers used in the triple procedure. Ophthalmology 1985, 92: 1561-6.

${ }^{14}$ Crawford GJ, Stulting RD, Waring GO, et al.: The triple procedure: analysis of the outcome, refraction and intraocular power calculation. Ophthalmology 1986, 93: 817-24.

${ }^{15}$ MacDonald HR and Irvine AR: Light-induced maculopathy from the operating room microscope in extracapsular extraction and intra-ocular lens implantation. Ophthalmology 1983, 90: 945-7.

${ }^{16}$ Kirkness CM and Weale RA: Does light pose a hazard to the macula in aphakia? Trans Ophthalmol Soc UK 1985, 104: 699-702.

${ }^{17}$ Sliney DH and Armstrong BC: Radiometric evaluation of surgical microscope lights for hazards analyses. Appl Optics 1986, 25: 1882-9. 